\title{
LE FRONTIERE DEL CORPO. IL VELO E L'IDENTITÀ FEM- MINILE NELLA SOCIETÀ MULTICULTURALE
}

\author{
Las fronteras del cuerpo. El velo y la identidad femenina en la sociedad multicultura. \\ The borders of the body. The veil, and female identity in multicultural society
}

Antonella Cagnolati Università degli Studi Foggia a.cagnolati@unifg.it

Fecha de recepción: 8-II-2012 Fecha de aceptación: 21-II-2012

Riassunto: La categoria di genere ed il concetto di multiculturalismo possono convivere? Se lo chiedeva già a metà degli anni Novanta Susan Moller Okin, dando origine ad un dibattito che oggi appare di scottante attualità in conseguenza delle polemiche sul velo. In Francia la discussione procede con toni aspri che rivelano la difficoltà di far coincidere teorie libertarie con modelli comportamentali che suscitano ostilità, rancore, diffidenza. Nella realtà la diatriba sul velo nasconde altri significati che rivelano la volontà di marcare con confini precisi l'identità, sia da un parte che dall'altra del confine socio-antropologico. La relazione vuole indagare dietro le prese di posizione per comprendere quali siano i nodi del contendere e per quale motivo le distinzioni e la xenofobia passino ancora una volta sul corpo delle donne, intesi come una trincea per la difesa di valori in opposizione.

Parole chiave: Velo, corpo, donne, genere, multiculturalismo, identità.

RESUMEN: ¿La categoría de género y el concepto de multiculturalismo pueden vivir uno al lado del otro? se preguntaba a mediados de los años noventa Susan Moller Okin, dando lugar a un debate de gran actualidad como resultado de la controversia sobre el velo. En Francia la discusión se desarrolla con tonos ásperos que revelan la dificultad que existe para conciliar las teorías libertarias con los patrones de conducta que despiertan la hostilidad, el resentimiento, la desconfianza. De hecho, la controversia sobre el velo, esconde otros significados que revelan la voluntad de marcar la identidad con límites claros de un lado y del otro de la frontera socio-antropológica. El artículo tiene como objetivo investigar acerca de las posiciones adoptadas en Europa sobre el velo con el fin de comprender cuáles son los 
nudos de la controversia y por qué las diferencias y la xenofobia pasan una vez más por el cuerpo de la mujer, entendido como una trinchera para la defensa de valores en oposición.

Palabras clave: Velo, cuerpo, mujeres, género, multiculturalismo, identidad.

ABSTRACT: The category of gender and the concept of multiculturalism can live together? In the mid-nineties Susan Moller Okin began questioning about it, giving rise to a debate that now seems highly topical as a result of the controversy over the veil. In France, the discussion proceeds with harsh tones that reveal the difficulty of reconciling liberal theories with behavioral patterns that arouse hostility, resentment, mistrust. In fact, the controversy over the veil hides other meanings which reveal the will to mark the female identity with sharp boundaries, as a part of the socio-anthropological borders over women's bodies. The report aims to investigate behind the positions taken, in order to understand which are the nodes of the dispute and why difference and xenophobia pass once again through the body of women, seen as a trench for the defense of values in deep opposition.

Keywords: Veil, body, women, gender, multiculturalism, identity.

\section{LE FASI DEL DIBATTITO}

Intendo affrontare nel mio saggio la tematica del contrasto tra l'idea di libertà così come è stata espressa e progressivamente affermata nelle carte dei diritti e nei testi giuridici, patrimonio della stessa radice culturale europea, e la prassi del multiculturalismo, come viene prendendo forma nella quotidianità delle esistenze delle donne immigrate, per le quali le teorie del liberalismo spesso nulla hanno a che vedere con le pratiche di annichilimento di coscienze, identità e corpi.

La questione è complessa e cercherò di rintracciare quindi anche le varie tappe di un progressivo confronto e irrigidimento tra diritti e culture, tra ideali e comportamenti, tra valori postulati in astratto e azioni sempre più efferate di violenza ed oppressione nei confronti delle donne di cui la cronaca ci riporta con fredda laconicità l'allarmante statistica.

Per meglio procedere nell'argomentazione, sarà utile porci alcune domande: quale partita si sta giocando sul corpo delle donne musulmane? Per quale motivo le muslima sono oggetto di tanta attenzione nell'Europa attuale? Il dibattito sul «velo» ${ }^{1}$ (termine assolutamente fuorviante) è reale oppure

$1 \mathrm{Il}$ termine «velo» è chiaramente di più immediata fruizione (dal punto di vista propagandistico) e comprensibile al vasto pubblico, piuttosto che la differente terminologia che definisce con notevoli varianti le particolarità dell'abbigliamento femminile. Forse dovremmo 
si tratta di un pretesto? Quale modello ideologico si cela dietro la battaglia per il ritorno alla tradizione, sia negli stati europei che nel Medio Oriente? Infine, in che orizzonte teorico si colloca la diatriba su genere e multiculturalismo? Dobbiamo procedere dunque ad un breve excursus che ci dia contezza del dibattito, intenso, profondo, a volte estenuante, in corso negli ultimi anni: partiamo quindi dalla cronaca più recente e andiamo poi a ritroso nel tempo.

Il 4 giugno 2009 il presidente degli Stati Uniti Barack Obama pronunciava uno storico discorso ${ }^{2}$ al Cairo con il quale la politica americana intendeva inaugurare una nuova stagione nei rapporti con il mondo arabo moderato. Nel suo lungo eloquio, ricco di richiami ideali e di proiezioni verso un futuro di relazioni collaborative, Obama toccava alcuni punti di notevole rilevanza per il tema che mi appresto a discutere: il primo, la libertà di professare la propria religione, il secondo la difesa dei diritti delle donne, non senza evidenti contraddizioni, frutto e conseguenza in larga misura di una visione multiculturalista.

Affermando che «il governo degli Stati Uniti si era costituito parte civile per proteggere il diritto delle donne e della ragazze di vestire l'hijab, e per punire coloro che vorrebbero negarlo» ${ }^{3}$, Obama stava implicitamente appoggiando la teoria che vede nell'abbigliamento delle donne musulmane soltanto il rispetto di una tradizione culturale che non si pone in contrasto con la laicità della nazione nella quale queste donne si trovano a vivere. Nella parte centrale del discorso le argomentazioni del presidente prendevano un tono più programmatico che si sustanziava in ben sette punti chiave della politica americana verso il mondo musulmano. Fra questi il sesto, relativo ai diritti delle donne, si focalizzava sul concetto di uguaglianza: Obama soste-

ricordare che la tradizione cristiana durante il rito della preghiera prevedeva il velo per le donne, secondo quanto afferma Paolo in 1 Corinzi, 11, 5, 13. Per un primo approccio alla questione si veda: LEILA AHMED, Women and gender in Islam. Historical roots of a modern debate, London, Yale UP, 1992; STEFANIA BARTOLONI (a cura di), A volto scoperto. Donne e diritti umani, Roma, manifestolibri, 2002; GIULIANA SGRENA, Il prezzo del velo, Milano, Feltrinelli, 2008; ÀNGELES RAMÍREZ, La trampa del velo. El debate sobre el uso del pañuelo musulmán, Madrid, Libros de la Catarata, 2011; WASSYLA TAMZALI, El burka como excusa. Terrorismo intelectual, moral y religioso contra la libertad de las mujeres, Barcelona, Saga Editorial, 2011.

2 Il discorso pronunciato da Barack Obama all'Università del Cairo (Cairo speech) ha rappresentato una pietra miliare nella politica estera americana post-11 settembre. Per il testo ufficiale completo si consulti www.nytimes.com/2009/06/04/us/politics/04obama.text. $\underline{\mathrm{html}}$ [9 febbraio 2012]; il video è visibile su www.youtube.com/watch?v $=\mathrm{B}$ 889oBKkNU. 3 www.nytimes.com/2009/06/04/us/politics/04obama.text.html (p. 2) [9 febbraio 2012]. 
neva di non essere d'accordo «con il punto di vista di alcuni in Occidente secondo i quali una donna che sceglie di coprire la sua capigliatura è in qualche modo meno uguale» ed aggiungeva che «egli rispetta quelle donne che scelgono di vivere le loro esistenze secondo ruoli ispirati dalla tradizione. Ma ciò dovrebbe rappresentare la loro scelta» ${ }^{4}$.

Il discorso di Obama ${ }^{5}$ è stato variamente commentato sia dai giornalisti americani che arabi, i quali hanno apprezzato la volontà di aprire un dialogo più aperto e costruttivo con il mondo arabo, necessità ampiamente avvertita dopo l'era Bush, rimarcando tuttavia con accenti entusiasti più la volontà di spingere verso il progresso, l'innovazione, l'istruzione ovviamente pagati con i proventi garantiti dalla vendita del petrolio che non sul fronte dei diritti umani, terreno quanto mai insidioso considerata la permanenza di una enclave come la prigione di Guantanamo dove sussiste una palese violazione dei più basilari diritti.

A maggior ragione, il passaggio sulla necessità di mantenere e rispettare la tradizione per quanto riguarda l'altra metà del mondo è stato interpretato in vario modo, a seconda dei punti di vista dei commentatori: se per alcuni si è trattato di un positivo ossequio alla cultura islamica, dall'altro le femministe arabe (algerine e marocchine) hanno criticato l'accondiscendenza verso prassi consuetudinarie palesemente lesive della volontà di scelta delle donne. In un sistema patriarcale in cui la decisione del pater familias è ritenuta superiore alla legge, è evidente che non sussiste possibilità alcuna per le donne di scegliere della loro vita, del loro futuro, della loro identità.

In realtà, Obama ben rappresenta con le sue parole il punto di vista del multiculturalismo imperante nello Zeitgeist del Terzo millennio, una teoria che mostra da tempo ormai non solo le sue chiare e pericolose contraddizioni, ma vede aprirsi al suo interno vistose crepe dovute alle prassi più che a meri scontri dottrinali alimentati dal mondo accademico oppure elaborati da ristretti gruppi di intellettuali.

Vediamo in maniera più dettagliata le prese di posizione conseguenti alle parole di Obama. Fin dall'inizio di ottobre 2009 la massima autorità religiosa dell’Università di al-Azhar lo sceicco Mohamed Tantawi ${ }^{6}$ si pronunciava

4 www.nytimes.com/2009/06/04/us/politics/04obama.text.html (p. 7) [9 febbraio 2010]. 5 news.bbc.co.uk/2/hi/middle_east/8083171.stm [10 febbraio 2010].

6 Mohamed Tantawi (1928-2010) ha ricoperto l'importante incarico di grande imam dell'Università Coranica Al Azhar del Cairo, il più antico istituto accademico religioso del 
apertamente contro il niqab ${ }^{7}$ per le studentesse e affermava che l'indumento doveva essere tolto in casi particolari, dal momento che era da ritenersi solo una prassi tradizionale, con nessuna base teologica nella fede musulmana ${ }^{8}$. La decisione suscitava violente polemiche al punto che non solo sui giornali egiziani ma perfino sull'emittente Al-Jazeera comparivano interviste rilasciate da autorevoli personaggi, infuocati commenti, accesi dibattiti' fino a che l'Alta Corte per gli affari amministrativi del Cairo il 3 gennaio 2010 si pronunciava a favore della decisione governativa di bandire il niqab durante gli esami all'interno degli spazi pubblici dell'università.

Ciò che mi pare interessante rilevare è la esplosione di rabbia di molte studentesse che accusavano apertamente Tantawi di aver «infranto i loro diritti» ${ }^{10}$. Ma la polemica nascondeva ben altre finalità: il niqab è ritenuto un abbigliamento tipico delle seguaci del wahabismo, ovvero dell'islam più fanatico ed estremista. Non a caso è diffuso (e obbligatorio) in molti paesi arabi del Golfo Persico, tra i quali l'Arabia Saudita, lo Yemen, il Bahrain, ed è al contrario osteggiato dai paesi arabi più moderati. Di conseguenza la vexata quaestio relativa al niqab rivela una contesa ben più aspra, dai toni sia religiosi che giuridici, sulla visibilità immediata delle penetrazione dell'islam radicale in nazioni che invece aderiscono ad una concezione più mitigata della fede islamica e che temono fortemente l'avanzata del fanatismo. L'esempio più evidente viene certamente dall'Egitto, paese nel quale ogni ricorso contro la legge che vieta il niqab è stato respinto ${ }^{11}$, ma anche dalla Siria che, in silenzio e senza troppo clamore, ha bandito dallo spazio pubblico delle università il controverso capo d'abbigliamento ${ }^{12}$, sia per le docenti che per le studentesse.

mondo arabo.

7 Il niqab è l'abito nero che copre completamente il corpo, corredato di un velo che serve a nascondere il viso, ad eccezione di una piccolissima apertura per gli occhi. Cfr. www. muhajabah.com/niqab-index.htm, sito sul quale si può reperire una distinzione dettagliata fra le ragioni della proibizione e della libertà di scelta in merito all'abbigliarsi con il niqab.

8 Gregg Carlstrom, «Tantawi takes aim at the niqab», in The Majlis, www.themajlis.org/ tag/niqab [10 febbraio 2012].

9 english.aljazeera.net/news/middleeast/2009/10/200910771027899622.html [10 febbraio 2010].

10 www.islamonline.net/servlet/Satellite?c=Article_C\&cid $=1162385844634 \&$

febbraio 2010].

11 english.aljazeera.net/news/middleeast/2010/01/201013195657854466.html [10 febbraio 2012]

12 «Le universitarie e le docenti degli atenei siriani, pubblici e privati, non potranno più indossare il velo integrale, il burqa o il più diffuso niqab che lascia scoperti soltanto gli occhi, perché contrario ai valori accademici. La direttiva del ministero dell'Istruzione si inserisce nel solco di analoghe iniziative nei Paesi mediorientali ed europei», «Siria, burqa 
Mentre dunque si fa più chiara la linea di demarcazione che passa dall'accettazione o dal rifiuto del niqab nei paesi arabi moderati, altrove si può andare dall'intransigenza talebana del burqa che rende la donna un fantasma, al mascheramento del corpo dovuto all'hijab, abito assai più presente sul suolo europeo $^{13}$. Ed è l'Europa il nuovo scenario in cui la discussione sul «velo» assume toni altrettanto accesi ed allarmanti ${ }^{14}$. Per comprendere che cosa stia avvenendo e come la tradizione giuridica europea si stia confrontando con le conseguenze della massiccia immigrazione, dobbiamo torniamo ancora una volta alle reazioni suscitate (questa volta in Europa) dal Cairo speech.

\section{STABILIRE CONFINI VISIBILI: IL CASO DELLA FRANCIA}

La nazione che si è sentita maggiormente colpita in negativo è stata la Francia e i vari distinguo non si sono fatti attendere. In un lungo editoriale ${ }^{15}$, Le Figaro criticava senza infingimenti le affermazioni del presidente, rimarcando come per ben «tre volte, M. Obama [avesse] preso la difesa del velo islamico, durante il suo discorso»: in nome della laicità, il giornale rimarcava

e niqab banditi dalle università», in www.ntnn.info/it/articles/siria-burqa-e-niqab-banditidalle-universita.htm [10 febbraio 2012].

13 Cfr. MAHMUD AKIF, La Femme dans le grandes civilisations et perspectives musulmanes, Paris, Essalam, 2006 (in particolare il cap. 2 La femme en Islam, pp. 53-91); ABDERRAZAK MAHRI, La femme dans le Coran, Paris, Maison d'Ennour, 2006 (in particolare sul velo pp. 145-151).

14 «Il Senato spagnolo ha approvato a sorpresa una mozione per chiedere al Governo di Jose Luis Rodriguez Zapatero di imporre il divieto di indossare il burqa in tutti i luoghi pubblici, anche in strada. Il testo ha ottenuto 131 voti favorevoli, tra cui quelli del Partito Popolare di Mariano Rajoy, dei nazionalisti catalani di Convergenza e Unione (CiU) e dell'Unione del Popolo Navarro. I voti contrari sono stati invece 129. L'approvazione della mozione arriva mentre in Catalogna è in corso un dibattito sull'opportunità di vietare il velo integrale nei luoghi pubblici. Nella regione nord orientale della Spagna sono già tre le municipalità, tra cui quella di Barcellona, che hanno imposto il divieto per le donne musulmane di indossare il burqa negli edifici pubblici. I socialisti di Zapatero avevano inizialmente stretto un accordo con il CiU e la Sinistra Repubblicana di Catalogna per votare no alla mozione, sostenendo che le leggi esistenti sono già sufficienti, ma i nazionalisti catalani hanno poi dato il loro sostegno al partito di Rajoy. La mozione richiede all'esecutivo di «utilizzare tutte le facoltà dell'ordinamento giuridico» per «regolamentare il divieto di utilizzare in pubblico il burqa e il niqab, per garantire l'eguaglianza, la libertà e la sicurezza». Il burqa nasconde completamente il corpo della donna, con una retina in corrispondenza degli occhi, che però rimangono non visibili. Il niqab copre invece il corpo fino alle ginocchia e lascia gli occhi scoperti. Gli oppositori a queste misure fanno notare che pochissime donne in Spagna indossano il velo integrale», «Burqa e niqab, Senato approva mozione per vietarlo in luoghi pubblici», in www.mondomistery.com/?p=120 [10 febbraio 2012].

15 «Hijab: Obama égratigne la France», in http://www.lefigaro.fr/flashactu/2009/06/04/01011-20090604FILWWW00594-hijab-obama-egratigne-la-france.php [11 febbraio 2012]. 
come la Francia avesse vietato fin dal 2004 con una legge ${ }^{16}$ ad hoc qualsiasi ostentazione di simboli religiosi e dunque le parole di Obama risuonavano nella stupenda sala dell'Università del Cairo come un pesante $j$ 'accuse nei confronti della politica assimilazionista intensivamente praticata dal governo francese. L'attacco di Le Figaro, successivo al Cairo speech, diventava sempre più preciso e dettagliato a partire dalla metà del giugno 2010: i comportamenti adottati dalla Francia in materia di prassi religiosa vengono suffragati da altri articoli che legittimano sempre le scelte improntate alla massima laicità. Così notizie dal Canada, dal Belgio, dai Paesi Bassi si fanno sempre più frequenti sulle pagine del giornale con l'esplicito intento di criminalizzare atti compiuti da appartenenti alla fede islamica: per esempio la notizia ${ }^{17}$ della condanna all'ergastolo di un padre che nel 2007 aveva ucciso la figlia di 16 anni colpevole di non voler portare il velo viene comunicata con particolare enfasi e compiacimento, quasi a voler indicare come il percorso intrapreso dalla autorità francesi sia del tutto corretto, giusto e apprezzato dalla nazione. Le Figaro segue con attenzione l'iter della legge al parlamento belga ${ }^{18}$ in cui si prevede di bandire burqa e niqab e si dà notizia dell'avvenuta approvazione. Viene pubblicato con grande enfasi un reportage curato da Nadjet Cherigui e Axelle de Russé intitolato «Enquête sur l'islam radical en France» ${ }^{19}$ che

16 Loi 2004-228, 15 mars 2004. Sulla discussione in merito al velo, si veda ALESSANDRA MARCHI, La Francia e l'islamofobia, Jura Gentium, VI, 2010, 1, in www.juragentium.unifi.it/ topics/islam/it/marchi.htm [6 febbraio 2012].

17 http://www.lefigaro.fr/flash-actu/2010/06/17/97001-20100617FILWWW00377-canada-il-tue-sa-fille-qui-refuse-le-hidjab.php [11 febbraio 2012].

18 All'inizio di aprile 2010 la commissione Affari interni della Camera belga ha approvato all'unanimità il bando del velo integrale. Il Parlamento ha votato il 22 aprile e dunque il Belgio è il primo paese europeo ad avere una norma così restrittiva. Il testo prevede una multa da 15 a 25 euro, cui potrà essere aggiunta la pena del carcere fino a sette giorni, per «chi si presenterà in uno spazio pubblico con il volto coperto, del tutto o in parte, in modo tale da impedirne l'identificazione». Le donne musulmane non potranno indossare né burqa né niqab per le strade, nei parchi, in ospedali e scuole, sugli autobus e in tutti i luoghi pubblici, compresi gli alberghi. La legge è stata presentata dalla maggioranza (liberali e cristianodemocratici) e sostenuta dai socialisti e dalla destra. ELIANA DI CARO, «Il Belgio mette al bando burqa e niqab», http://www.ilsole24ore.com/art/SoleOnLine4/Mondo/2010/04/ belgio-burqa-niqab-bando.shtml [6 febbraio 2012].

19 Ce reportage est un document. Un témoignage exceptionnel. Pour la première fois, en plein débat sur l'identité française, au moment même où le port du voile divise l'ensemble de la communauté musulmane, des femmes salafistes ont accepté de s'expliquer. De se livrer comme jamais et d'ouvrir leurs portes à des journalistes. Pour aller aussi loin, pour plonger ainsi au coeur de l'islam radical de France et gagner la confiance de ces femmes qui ne sortent que très raramente de chez elles, Nadjet Cherigui et Axelle de Russé ont négocié des semaines», in http://www.lefigaro.fr/actualite-france/2009/11/06/0101620091106ARTFIG00587-enquete-sur-l-islam-radical-en-france-.php [10 febbraio 2012]. 
desta scalpore per le notizie che emergono sulla diffusione dell'estremismo di matrice salafista in Francia, in particolare l'acritica accettazione dei dettami più rigidi della sharia in materia di abbigliamento da parte delle donne. Il reportage è corredato da foto che sembrano scattate in qualche angolo di periferia di una grande metropoli araba e non certamente a Parigi.

Si sta con tutta evidenza lanciando una vasta campagna mediatica: se non è ovviamente possibile che il presidente americano sia il bersaglio esplicito, visto il grande prestigio di cui egli gode in Europa, si va per lo meno ad inficiare il suo giudizio sulla validità del velo e sulla libertà di culto ${ }^{20}$. La Francia si sente attaccata e risponde con i fatti, buttati in prima pagina quasi a voler replicare ai proclami libertari di Obama con la brutalità degli episodi più cruenti tratti dalla cronaca, e diventati ormai un malinconico rituale de profundis.

Vi è tuttavia un passaggio cruciale che vorrei sottolineare, ovvero la complessità socio-antropologica di un concetto quale è attualmente l'idea di nazione, peraltro ormai obsoleto dal punto di vista culturale come Bauman ${ }^{21} \mathrm{e}$ Maloouf $\mathrm{f}^{22} \mathrm{ci}$ hanno sapientemente spiegato. Il lessico della politica utilizza ormai il termine «nazione» in un'accezione che spesso non ha più alcuna aderenza con la realtà del nostro vissuto quotidiano ed in particolare adotta uno schema logico-argomentativo che si fa strumento di contrapposizione palese tra un «noi» e un «loro». Se non si può agire in termini di netta ostilità, dal momento che esistono i diritti a tutela di «tutti», pare più semplice ed immediato dare battaglia sul fronte dei comportamenti da criticare come antiquati, lesivi, offensivi nei confronti dei valori nazionali.

Ma di quale nazione stiamo parlando? In una ricerca sull'identità «francese» lanciata per esplicita volontà del presidente Sarkozy nell'estate del 2009 emerge con sconcertante allarme come i francesi non sappiano più realmente chi sono, quali siano le loro radici culturali, le fonti del loro immaginario collettivo. I risultati dell'inchiesta sono talmente dissacranti che spingono l'acceleratore dell'Eliseo sulla volontà di far riemergere gli ideali puramente francesi e di lanciare una campagna in difesa dell'identità (il vento di destra

20 Sul dibattito relativo all'uso del velo, cfr. il corposo reportage apparso su «Le Monde Magazine» dal titolo Niqab Un autre regard il 30 gennaio 2010 che mette a confronto le posizioni a favore e contro il velo, ascoltando la voce delle donne musulmane.

21 ZYGMUNT BAUMAN, Intervista sull'identità, Bari-Roma, Laterza, 2009.

22 AMIN MAALOUF, L’identità, Milano, Bompiani, 2005. 
soffia forte...). In un illuminante articolo pubblicato su «Il Corriere della Sera», Bernard Henry Levy scriveva che vi sono almeno tre buoni motivi per rifiutare un simile dibattito così orientato politicamente,

forzato, inquadrato, delimitato da ogni lato, controllato. È perché si ama il dibattito, è perché si crede che non esistano idee preconcette, opinioni, certezze che non meritino di essere sconvolte dal benefico stimolo di un libero dibattito, che bisogna rifiutare questo falso dibattito, questa caricatura di dibattito, dove viene intimato a sessanta milioni di cittadini infantilizzati di consegnare il loro compito al Grande Esaminatore, il quale suonerà il campanello che segna la fine, non della ricreazione, ma del dibattito. Il secondo problema sta nel fatto che tale dibattito di Stato nasce da uno Stato che - circostanza aggravante - è il primo, nella nostra Storia, ad aver inventato quell'eresia repubblicana che è il «ministero dell'identità nazionale e dell'immigrazione». [...] Ma, al tempo stesso, le parole hanno una storia. I linguaggi, un inconscio. E le libere associazioni che essi generano sono come bombe che esplodono nei cervelli: anche e soprattutto quando gli artificieri non l'hanno previsto né voluto. Tutto comincia con la piccola «e» del «ministero dell'identità nazionale e dell'immigrazione»: con l'annuncio fragoroso di una compresenza, nella stessa concatenazione significante, dell'idea che esista un malessere nella civiltà nazionale e un problema legato al nostro modo di gestire l'immigrazione. Ed ecco che il movimento è lanciato! Un passo di più ed è il sottosegretario alla Famiglia, Nadine Morano che, all'unisono con $i$ discorsi nauseabondi che ormai si odono in tutte le prefetture, descrive i giovani musulmani come cattivi francesi riluttanti a integrarsi. Sono sbandamenti? No. Sono effetti strutturali di uno scenario impiantato tre anni fa. Meccanismo di un discorso che non poteva operare senza escludere, stigmatizarar, alimentare le tensioni e gli odii. Liberazione di una parola xenofoba, se non razzista, che i cittadini di destra e di sinistra volevano all'unanimità contenere, ma che improvvisamente si trova ad essere benedetta da tutti gli organi di un apparato di Stato democratico che perde la testa. C’è poi un terzo problema concernente l'uso che vien fatto della nozione stessa di identità. Fin dall'inizio, ho suggerito che, identità per identità, se esiste un'identità che per un francese è un problema, se esiste un'identità in panne, questa è l'identità europea. Ma la verità vera è che il concetto stesso di identità era filosoficamente una trappola... ${ }^{23}$

23 BERNARD HENRY LEVY, La Francia e l'identità nazionale, un dibattito che Sarkosy deve chiudere, «Il Corriere della Sera», 8 gennaio 2010, p. 12. 
Parole sconvolgenti in una paese che conta ormai milioni di immigrati e cittadini di seconda e terza generazione, come ampiamente testimoniato dalla composizione multietnica dei Blues, i giocatori della nazionale francese di calcio fin dal Campionato del mondo del 2006, di cui tutti credo abbiamo triste memoria, se non altro per le parole offensive nei confronti di un simbolo della perfetta integrazione come Zinedine Zidane.

Il grande dibattito si iscrive nella politica fortemente voluta dal presidente francese finalizzata a ristabilire una coesione nazionale oppure si tratta di sottrarre alle destre il monopolio della questione xenofoba sulla quale il Fronte nazionale costruisce il suo consenso elettorale? Probabilmente la risposta si rivela assai più complessa e multiforme ${ }^{24}$ : la Francia sta vivendo un momento di rilevante crisi identitaria le cui cause sono da ricercare sia nel fallimento del modello economico attuale, sia nella palese insufficienza del progetto di integrazione sociale. È ancora devastante l'eco delle rivolte nelle banlieues parigine nel 2005, evento quanto mai traumatico a livello simbolico, che ha visto protagonisti i figli della cosiddetta «seconda generazione», spinti dalla disperazione di non avere un futuro da cittadini ma solamente da sfruttati, oppure il dibattito suscitato dalla ricerca di Attias e Wolf ${ }^{25}$ relativa ai meccanismi di ascesa sociale per le terze generazioni.

Il mondo della cultura si è interrogato parimenti sull'idea di francité negli austeri palazzi dell'Institut Montaigne, mentre autorevoli intellettuali cercavano di declinare, ognuno secondo il proprio ambito disciplinare, il paradigma dell'identità francese. Dunque il dibattito resta aperto, in pieno fermento, vuoi per motivi meramente politici, vuoi anche per replicare e trovare soluzioni positive ad una crisi di crescita della società che potrebbe portare tuttavia ad una radicalizzazione dei comportamenti, ponendo in feroce antitesi les citoyens e les autres, senza ragionare sull'evidente verità che spesso i presunti «altri» sono nati sullo stesso suolo, parlano la medesima

24 Sulla complessa costruzione dell'altro come nemico e sulla demonizzazione dei simboli, si veda MONDHER KILANI, L'invenzione dell'altro, Bari, Dedalo, 1997 (in particolare il cap. XI dal titolo La Francia e il velo islamico. Universalismo, comparazione, gerarchia, pp. 265-306); ERDMUTE HELLER, HASSOUNA MOSHABI, Dietro il velo, Roma-Bari, Laterza, 1996 (in particolare cap. X dal titolo Il velo e le proibizioni dell'islam); ANNAMARIA RIVERA, La guerra dei simboli. Veli postcoloniali e retoriche dell'alterità, Bari, Dedalo, 2005.

25 Si tratta della ricerca compiuta da due sociologi francesi relativa ai figli degli immigrati: CLAUDINE ATTIAS-DONFUT, FRANÇOIS-CHARLES WOLF, Le destin des enfants d'immigres: un desenchainement des generations, Paris, Stock, 2009. 
lingua, vanno a scuola con i propri figli, si riconoscono come tifosi della stessa nazionale.

Non si può negare che il panorama attuale dei flussi migratori possa dare esiti nefasti come il recente volume di Michèle Tribalat che giunge ad ipotizzare, manipolando abilmente le cifre fornite dalla statistiche, come il governo attui una politica di censura sulla vera entità numerica della presenza di immigrati sul territorio francese ${ }^{26}$.

È parimenti in corso una ripensamento globale sulla parola-chiave sulla quale poggia l'intera impalcatura della struttura statale francese: la laicità, vista come effettiva discriminante tra uno stato che ha già eliminato qualsiasi simbologia religiosa dallo spazio pubblico, ed una entità che invece fa della propria appartenenza ad una fede un costrutto identitario sul quale non si è disposti a transigere: si comprenderà facilmente che il dialogo sia quanto mai ostico e foriero di incomprensioni, fraintendimenti, manipolazioni.

Per quale motivo si pone tanta enfasi in questo argomentare mai scevro di venature polemiche? In quale contesto politico e culturale si sta collocando la battaglia per la laicità della nazione? E perché la posta in gioco passa sul corpo delle donne?

Alla stregua di molti altri passaggi storici di grande rilevanza, in occasione di crisi culturali o di pericoli all'orizzonte, la frontiera è sempre e comunque quella che passa dal corpo femminile, un corpo che viene descritto nella sua fisiologia, di cui si scrutano e si narrano con dovizia di particolari le perverse patologie, fisiche o psicologiche, un corpo che può subire torture e violenze in nome di un'ideologia che annichilisce le donne come soggetti di diritto ${ }^{27}$.

26 MICHÉLE TRIBALAT, Les yeux grands fermés. L'immigration en France, Paris, Denoël, 2010.

27 Sulla posizione politica e culturale delle donne musulmane si veda RUBA SALIH, $M u-$ sulmane rivelate. Donne, islam, modernità, Roma, Carocci, 2008; ANNA VANZAN, Le donne di Allah. Viaggio nei femminismi islamici, Milano, Bruno Mondadori, 2010. 


\section{Genere vs multiculturalismo}

Le donne musulmane, che la sociologa marocchina Fatema Mernissi descriveva come coloro che portano la frontiera del desiderio tatuata sul corpo, rivelano, con la loro visibilità fisica e simbolica, con la loro corporeità, un'eccedenza che si vorrebbe negare, cancellare. In questa congiuntura il corpo delle donne acquisisce una funzione strategica fondamentale diventando per un verso il teatro delle battaglie per i diritti delle minoranze e il loro riconoscimento in Occidente, per un altro il luogo simbolico della conferma dell'impossibilità di tale riconoscimento. Oggetto di contesa, il corpo della donna musulmana diventa il campo di una doppia oppressione prescrittiva: il dover essere velata (secondo il rispetto dei dettami tradizionali propri della comunità di appartenenza) e il dover essere scoperta (secondo le prescrizioni della laicità di stato o in base agli imperativi dell'emancipazione femminile $)^{28}$.

Nella società contemporanea i conflitti culturali e normativi sempre più frequentemente coinvolgono le donne e tra le questioni che l'immigrazione mette in luce, in Italia come in altri paesi occidentali, quelle che riguardano la condizione delle donne sono tra le più pressanti e complesse. E giusto pretendere che le altre culture si conformino agli standard liberali di eguaglianza di genere? Oppure dovremmo rispettare le tradizioni, le credenze e le norme delle minoranze culturali presenti all'interno delle nostre comunità nazionali? Fino a che punto?

La questione venne affrontata dalla teorica politica Susan Moller Okin, nel saggio dal titolo provocatorio Il multiculturalismo è un male per le donne?, nel quale sosteneva la necessità di superare la formula multiculturalista tesa a garantire i diritti del gruppo delle minoranze per proteggere anzitutto i diritti delle donne ${ }^{29}$. L'analisi di Okin si è concentrata in particolare sulla posizione di Kymlicka ${ }^{30}$, assunto quale modello di tutte le teorie multiculturaliste che sostengono e cercano di implementare la difesa dei diritti di gruppo delle minoranze culturali. Il merito della provocazione di Okin è di avere evidenziato che non è così scontato che il multiculturalismo sia necessariamente il paladino di ogni differenza, ma che al contrario può darsi il caso

28 http://marginaliavincenzaperilli.blogspot.com/2009/08/lislam-fa-male-alle-donne-critica-del-multiculturalismo-e-ragioni-postcoloniali.html [28 gennaio 2012].

29 SUSAN MOLLER OKIN, Il multiculturalismo è un male per le donne?, in SUSAN MOLLER OKIN, Diritti delle donne e multiculturalismo, Milano, Raffaello Cortina Editore, 2007, pp. 3-22.

30 WILL KYMLICKA, La cittadinanza multiculturale, Bologna, il Mulino, 1999. 
che si riveli difficilmente conciliabile con determinate forme di differenza e di mancato riconoscimento di diritti.

È questo il caso delle rivendicazioni del multiculturalismo, e anzi sono molto probabili delle tensioni, per essere più precisi, fra il femminismo e un impegno multiculturalista per i diritti di gruppo delle minoranze. Questo accade per svariate ragioni, tra cui il fatto di confondere una condizione sociale e storicamente costruita, come l'appartenere e il riconoscersi in un determinato gruppo culturale, con una condizione biologica, l'essere donna; il non riconoscere o il riconoscere solo parzialmente che i diritti di gruppo sono spesso antifemministi; il non avere prestato sufficiente attenzione alle differenze che esistono all'interno di ogni gruppo, concentrandosi solo sulle differenze fra i gruppi; la totale disattenzione per le regole che i gruppi impongono sull'ambito della vita familiare o domestica dove frequentemente le donne vivono in una condizione di subalternità. Tutte ragioni che, nell'analisi di Okin, dimostrano la cecità del dibattito multiculturale nei confronti di una delle dinamiche centrali nella rivendicazione del riconoscimento di diritti speciali o privilegi culturali, il controllo delle donne. Okin osserva che nelle teorie multiculturali i diritti speciali vengono negati solo a quei gruppi che discriminano le donne in modo manifesto e formale, cioè che non riconoscono loro formalmente, dichiaratamente i diritti individuali liberali, mentre la pratica mostra che la discriminazione sessuale, anche se vietata a livello formale, può essere molto più diffusa. In particolare, Okin concentra la sua argomentazione sul nodo cruciale dei rapporti di potere all'interno della famiglia intesa non come ambito privato bensì come universo concentrazionario all'interno del quale vigono rapporti di forza assolutamente sbilanciati, in un puzzle composto da una viepiù rafforzata idea dell'onore, dalla proibizione per le donne nubili dell'esercizio di qualsiasi sessualità, di una palese negazione dell'identità e dell'autonomia ${ }^{31}$.

Come pare evidente, il confronto passa dunque attraverso una decostruzione dei paradigmi fondanti della società patriarcale che vigono all'interno della sfera privata in cui le donne si trovano a vivere, basati sulla prassi del controllo e del disciplinamento, e le logiche di una società liberale che si basa sui principi dell'universalismo ${ }^{32}$.

$31 \mathrm{Si}$ veda su tali tematiche, SOUAD SBAI, L'inganno. Vittime del multiculturalismo, Firenze, Cantagalli, 2010.

32 Cfr. ANNE PHILLIPS, Multiculturalism without Culture, Princeton, NJ, Princeton University Press, 2007; CARLO GALLI, L'umanità multiculturale, Bologna, il Mulino, 2008. 